\title{
Sellar Neurocytoma
}

National Cancer Institute

\section{Source}

National Cancer Institute. Sellar Neurocytoma. NCI Thesaurus. Code C155768.

A rare extraventricular neurocytoma (WHO grade II) of the hypothalamic-pituitary area.

(WHO 2017) 